\title{
A Parametric Device Study for SiC Power Electronics
}

\author{
Burak Ozpineci ${ }^{1,3}$ \\ burak@ieee.org \\ Leon M. Tolbert ${ }^{1,2}$ \\ Syed K. Islam ${ }^{1}$ \\ Md. Hasanuzzaman ${ }^{1}$ \\ sislam@utk.edu \\ mhasanuz@utk.edu \\ ${ }^{1}$ Department of Electrical and \\ Computer Engineering \\ The University of Tennessee \\ ${ }^{2}$ Oak Ridge National Laboratory \\ P.O. Box 2009 \\ Oak Ridge, TN 37831-6472 \\ ${ }^{3}$ Oak Ridge Institute for Science
and Education \\ Oak Ridge, TN 37831-0117
}

Knoxville, TN 37996-2100

\begin{abstract}
Materials and device researchers build switching devices for the circuits researchers to use in their circuits, but they rarely know how and where the devices are going to be used. The circuits people, including power electronics researchers, take the devices as black boxes and use them in their circuits not knowing much about the inside of the devices. The best way to design optimum devices is an interactive design where people designing and building the devices have a close interaction with the people who use them.

This study covers the circuit aspects of the SiC power device development. As a contribution to the above-mentioned interactive design, in this paper, the device parameters, which need to be improved in order to design better devices, will be discussed.
\end{abstract}

\section{INTRODUCTION}

Typically, power electronics researchers have to choose off-the-shelf power devices with the specifications best fit for their applications. They, usually, do not have a say about how they would like the device parameters be changed.

Materials and device researchers build switching devices for the power electronics researchers to use in their circuits, but they rarely know how and where the devices are going to be used.

As represented in Fig. 1, a "barrier" exists between the people who design and build power devices and the people who use them in their circuits and systems. Close interaction between the both sides of the barrier is needed to obtain the most performance for devices and systems. With this interaction, the design loop will be closed and the possibility for building application specific optimum power devices will arise.

Recently, a significant increase in the interest of silicon carbide ( $\mathrm{SiC}$ ) power devices has occurred because of their system level benefits. In the literature, $\mathrm{SiC}$ research is mainly concentrated on the materials and devices aspects $[1,2]$. Recently, more circuit applications [3, 4] are being published.

Prepared by the Oak Ridge National Laboratory, Oak Ridge, Tennessee 37831, managed by UT-Battelle for the U.S. Department of Energy under contract DE-AC05-00OR22725.

The submitted manuscript has been authored by a contractor of the U.S. Government under Contract No. DE-AC05-00OR22725. Accordingly, the U.S. Government retains a non-exclusive, royalty-free license to publish from the contribution, or allow others to do so, for U.S. Government purposes.

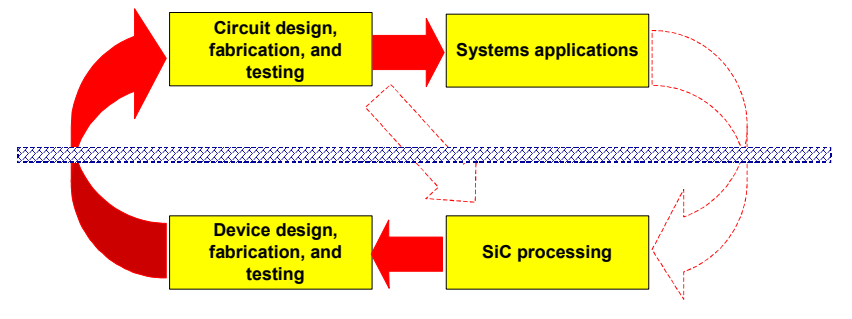

Fig 1. Closing the device design loop.

Moreover, the system level benefits of $\mathrm{SiC}$ are also being evaluated in some recent papers [5-7]. However, $\mathrm{SiC}$ power devices are still in their development stage; therefore, this is a good opportunity at this time to close the loop.

At Oak Ridge National Laboratory (ORNL), a team of materials, device, and power electronics researchers are working together with the University of Tennessee, Auburn University, and Vanderbilt University to build application specific optimum $\mathrm{SiC}$ power MOSFETs. This paper will summarize some of this work.

\section{APPLICATIONS}

This paper is a part of a study where system impact of $\mathrm{SiC}$ power electronics on hybrid electric vehicle (HEV) applications was investigated [5-8]. In the mentioned study, two HEV power converters were identified, modeled, and simulated to show the system level benefits of $\mathrm{SiC}$ power electronics quantitatively. The two selected applications were a dc-dc power supply and a traction drive.

The dc-dc power supply shown in Fig. 2 is an isolated fullbridge dc-dc converter, which is selected mostly because of its high frequency transformer, which provides isolation and additional taps in the secondary to feed more than one converter.

The main traction drive shown in Fig. 3 uses most of the power in an HEV when the vehicle is in motion. A traction drive consists of a battery feeding a three-phase induction machine through a three-phase inverter. Because of the cooling requirements of the power devices in the inverter, usually a large heatsink is required.

In an $\mathrm{HEV}$, any reduction in volume and weight of any component will benefit the efficiency of the vehicle. Because $\mathrm{SiC}$ devices can operate at higher temperatures and they have 


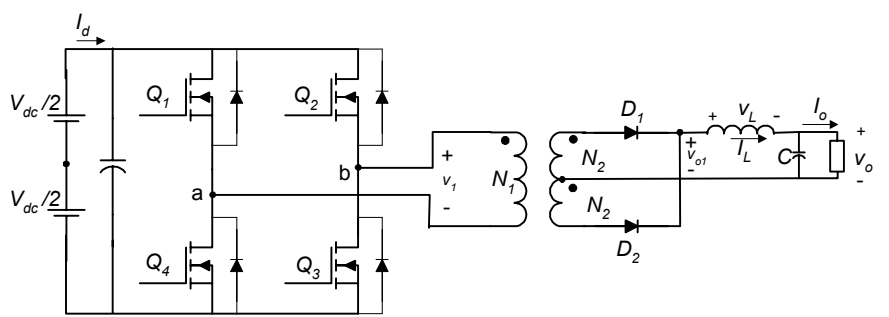

Fig 2. Isolated full-bridge step-down dc-dc converter.

lower losses, the heatsink volume and weight can be reduced if all $\mathrm{SiC}$ devices are used in all HEV power converters.

The simulation results of these converters have shown on average $30 \%$ decrease in weight and volume of the heatsink and a $5-10 \%$ increase in the efficiency. Improving the related device parameters can increase these further.

In the next two sections, these parameters will be identified for SiC Schottky diodes and MOSFETs and then necessary suggestions for improvement will be stated.

Note that all these modification suggestions also apply to $\mathrm{Si}$ devices, but the main focus of this study is given to $\mathrm{SiC}$ power devices.

\section{DIODES}

Some important diode parameters for power electronics systems are the breakdown voltage, on resistance, built-in voltage, peak reverse recovery current, and reverse recovery time.

\section{A. Conduction Loss Parameters}

1) Traction drive

A diode conduction loss expression for a traction drive inverter shown in Fig. 3 has been derived in [5], and it is repeated below for convenience.

$$
P_{\text {cond }, D 4}=I^{2} \cdot R_{D} \cdot \frac{\S}{\complement} 8 \frac{1}{3 \pi} M \cos \phi_{\mathfrak{I}}^{\cdot}+I \cdot V_{D} \cdot \frac{\S}{\complement 2 \pi}-\frac{1}{8} M \cos \phi_{\mathfrak{I}}
$$

where $I$ is the current through the diode,

$M$ is the modulation index for sinusoidal PWM,

$\phi$ is the power factor angle,

$R_{D}$ is the diode series resistance, and

$V_{D}$ is the diode built-in voltage.

This equation consists of two parts, loss associated with

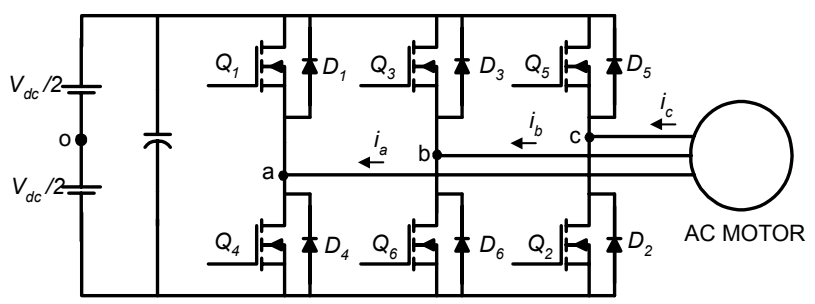

Fig. 3. Three-phase inverter driving an induction machine load. the on resistance, $R_{D}$ and loss associated with the built-in voltage drop, $V_{D}$. Diodes with lower $R_{D}$ and $V_{D}$ would be preferable, but these parameters depend on similar device parameters e.g. both of these parameters depend on the doping densities. Higher doping density means lower $R_{D}$ but higher $V_{D}$ and lower breakdown voltage, $B V$; therefore, both $R_{D}$ and $V_{D}$ cannot be lowered at the same time, i.e. a trade-off is required.

Consider a $4 \mathrm{H}-\mathrm{SiC}$ Schottky diode with a $B V$ of more than $500 \mathrm{~V}$ for a traction drive.

$$
\begin{aligned}
& B V \approx \frac{\mathcal{E}_{r} E_{c}^{2}}{2 q N_{d}}=\frac{1.3511 \times 10^{21}}{N_{d}}>500 \mathrm{~V}, \text { and } \\
& N_{d}<2.7 \times 10^{18} \\
& \text { where } \quad B V \text { is the breakdown voltage } \\
& \mathcal{E}_{r} \text { is the permittivity } \\
& E_{c} \text { is the critical electric breakdown field } \\
& q \text { is the electron charge } \\
& N_{d} \text { is the doping density }
\end{aligned}
$$

The maximum doping density value to sustain the chosen $B V$ is calculated above. The resistance value corresponding to this $N_{d}$ is the minimum $R_{D}$. It cannot be decreased with doping any further; however, the doping density can still be selected lower than this value, which would increase $B V$ and $R_{D}$, and decrease $V_{D}$. Then, the question is: Can modifying $V_{D}$ and $R_{D}$ decrease the conduction losses?

To answer this question, it is required to find how much changes in $R_{D}$ and/or $V_{D}$ will affect the conduction losses.

$$
I^{2} \cdot R_{D} \cdot \frac{\S 1}{\complement}-\frac{1}{3 \pi} M \cos \phi_{\mathrm{I}}>?<I \cdot V_{D} \cdot \frac{\S}{\complement 2 \pi}-\frac{1}{8} M \cos \phi_{\mathrm{I}}
$$

Rearranging terms and assuming $I \neq 0$,

$$
\begin{aligned}
& I \frac{\S \frac{1}{C 8}-\frac{1}{3 \pi} M \cos \phi_{\mathfrak{I}}^{\cdot}}{\S \frac{1}{\bigodot 2 \pi}-\frac{1}{8} M \cos \phi_{\mathfrak{I}}^{\cdot}}>?<\frac{V_{D}}{R_{D}} \\
& I \cdot f(M \cos \phi)>?<\frac{V_{D}}{R_{D}}
\end{aligned}
$$

$$
\text { where } \quad f(M \cos \phi)=\frac{\S \frac{1}{\S}-\frac{1}{3 \pi} M \cos \phi_{\overrightarrow{1}}}{\S \frac{1}{\complement 2 \pi}-\frac{1}{8} M \cos \phi_{\overrightarrow{1}}^{\cdot}},
$$

$M$ is the modulation index, which varies between 0 and $4 / \pi$ (square wave operation), and $\cos \phi$ is the power factor, which varies between 0 and 1 . The power factor of an induction machine is always lagging; for this example calculation, it is assumed to be 0.9 at rated load.

$$
0 \leq M \leq \frac{4}{\pi} \text { and } 0 \leq \cos \phi<0.9
$$

Then, 


$$
0 \leq M \cos \phi<\frac{3.6}{\pi}
$$

and $f(M \cos \phi)$ varies between 0.787 (no-load) and 0.215 (rated load) as shown in Fig. 4.

At first glance, it might seem that because the $I \cdot V_{D}$ multiplier is larger than the $I^{2} \cdot R_{D}$ multiplier at all $M \cos \phi$ values in Fig. 4, the $V_{D}$ losses should always be higher. This observation would have been true if and only if $V_{D}$ and the $I \cdot R_{D}$ product were equal. This, however, is not the case, and that is why, all three of these variables are included in (4) to find under what conditions, what part of the conduction losses is higher. The following example illustrates how to make use of (4).

For a particular hybrid electric vehicle traction drive, the rated peak machine current is 136.28A, which makes $I \cdot f(M \cos \phi)=136.28 \cdot 0.215=29.3 A$. Ignoring the off condition, the minimum device current is the magnetizing current, which is $71 \mathrm{~A}$. During the magnetizing current operation, the phase angle is almost $\pi / 2$ radians and the power factor is almost zero, then

\section{$I \cdot f(M \cos \phi)=71 \cdot 0.787=55.9 \mathrm{~A}$}

Considering (4), the following are some recommendations to maximize the efficiency of a $\mathrm{SiC}$ diode in a traction drive application:

1) If $29.3 \mathrm{~A}>\frac{V_{D}}{R_{D}}$,

then the $R_{D}$ losses are higher at all times, keep the doping density and $R_{D}$ constant because decreasing $R_{D}$ means decreasing $B V$, which would limit the device's application.

2) If $55.9 \mathrm{~A}<\frac{V_{D}}{R_{D}}$,

then the $V_{D}$ losses are higher at all times, decrease the doping density so that $V_{D}$ will be smaller.

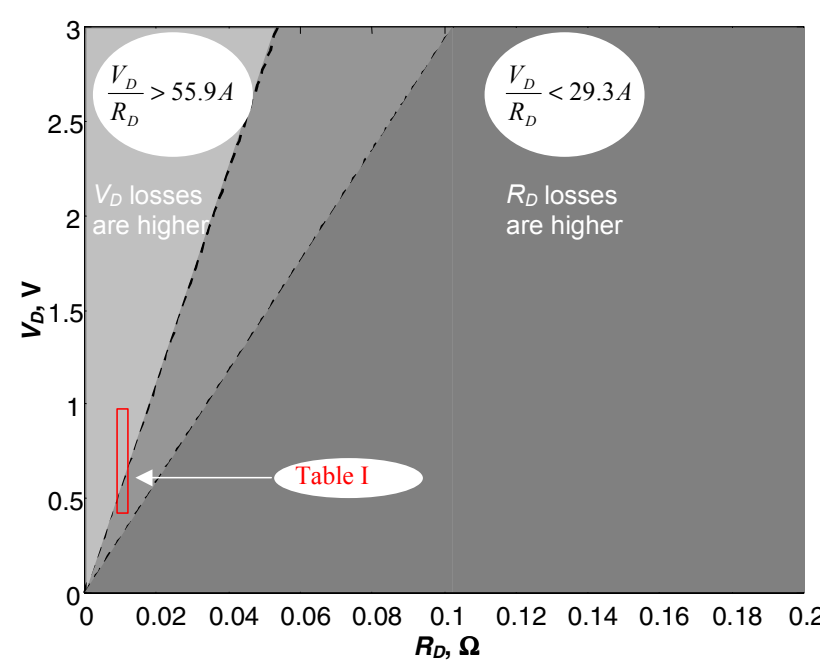

3) If $29.3 \mathrm{~A}<\frac{V_{D}}{R_{D}}<55.9 \mathrm{~A}$,

then the average current of operation will determine the recommended doping density as follows:

a) A drive working close to its rated current value uses the condition

29.3A $<\frac{V_{D}}{R_{D}}$, where $V_{D}$ losses are higher, decrease the doping density so that $V_{D}$ will be smaller.

b) A drive working at light current loads uses the condition

$\frac{V_{D}}{R_{D}}<55.9 A$, where $R_{D}$ losses are higher, keep the doping as it is because decreasing $R_{D}$ means decreasing $B V$, which would decrease the voltage blocking capability of the device.

Fig. 5 displays the above statements on an $R_{D}-V_{D}$ plane.

A commercial $\mathrm{SiC}$ Schottky diode $I-V$ characteristics are obtained at different temperatures. From these characteristics, $V_{D}$ and $R_{D}$ values of the diode are calculated. These values are tabulated in Table I and shown as a small rectangular area in

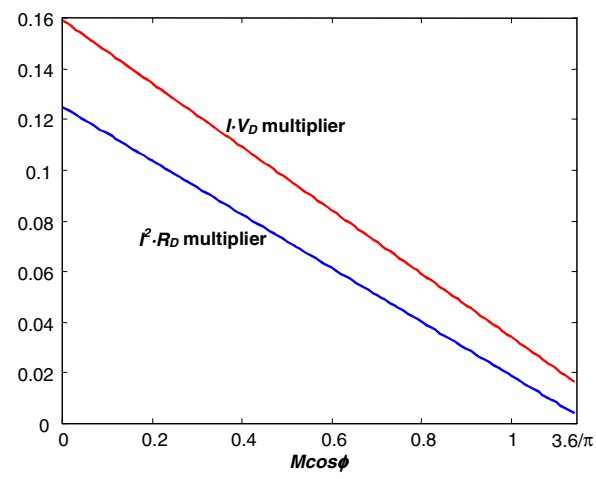

(a)

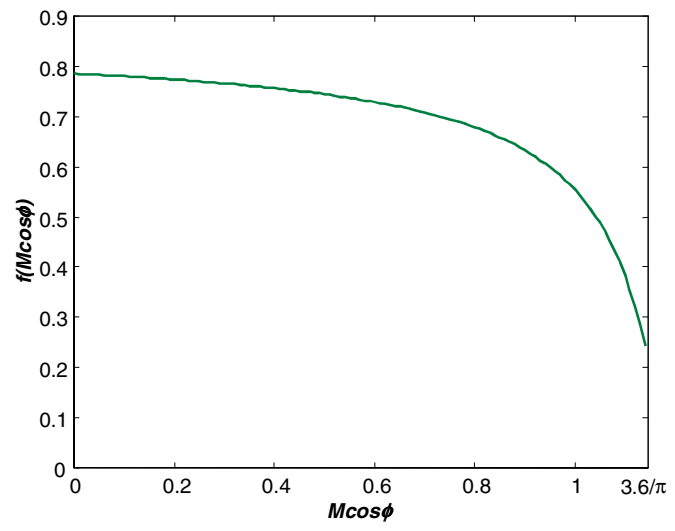

(b)

Fig. 5. The variation of $f(M \cos \phi)$ with $M \cos \phi$ (a) The denominator and the numerator of $f(M \cos \phi)$ vs. $M \cos \phi$ (b) $f(M \cos \phi)$ vs. $M \cos \phi$.

Fig 4 . The $R_{D}-V_{D}$ plane for the traction drive. 
TABLE I

SiC Diode Pwl Model Parameters and $V_{D} / R_{D}$ Ratio

\begin{tabular}{cccc}
\hline $\boldsymbol{T}_{\text {oven }},{ }^{\circ} \mathbf{C}$ & $\boldsymbol{R}_{\boldsymbol{D}}, \mathbf{m} \boldsymbol{\Omega}$ & $\boldsymbol{V}_{\boldsymbol{D}}, \mathbf{V}$ & $\boldsymbol{V}_{\boldsymbol{D}} / \boldsymbol{R}_{\boldsymbol{D}}, \boldsymbol{A}$ \\
\hline 27 & 4.2 & 1.07 & 254 \\
61 & 9.4 & 0.63 & 67 \\
82 & 10.3 & 0.56 & 55 \\
106 & 8.9 & 0.68 & 76 \\
129 & 10.0 & 0.59 & 59 \\
150 & 11.5 & 0.55 & 48 \\
174 & 11.7 & 0.55 & 48 \\
200 & 11.8 & 0.50 & 42 \\
250 & 12.1 & 0.48 & 40 \\
\hline
\end{tabular}

Fig. 5. Also shown in Table I is the corresponding $V_{D} / R_{D}$ ratios at different operating temperatures.

At temperatures up to and including $129^{\circ} \mathrm{C}$, the $V_{D} / R_{D}$ ratio is greater than $55.9 \mathrm{~A}$, therefore $V_{D}$ losses are higher. At the other temperatures, the ratio is between $29.3 \mathrm{~A}$ and 55.9A. The traction drive will operate close to the rated operation of the induction machine; therefore, consider the comparison with 29.3A. For all the other temperatures, the ratio is greater than $29.3 \mathrm{~A}$; thus, the $V_{D}$ losses are higher again.

As a conclusion for this case, if the doping concentration, $N_{d}$ for the $\mathrm{SiC}$ diodes in this study is decreased, then $V_{D}$ and the conduction losses decrease. The limit of this decrease is determined by the $V_{D} / R_{D}$ ratio.

Equation (4) can be used for any sinusoidal PWM application as long as the operation current, power factor, and modulation index information is available.

\section{2). Dc power supply}

The conduction loss expression for the isolated full-bridge dc-dc converter shown in Fig. 2 is as follows:

$$
P_{\text {cond }}=d\left(I_{D} \cdot V_{D}+I_{D}^{2} \cdot R_{D}\right)
$$

where $d$ is the duty ratio of the diode.

Using the same approach as in the previous subsection, the dominant losses can be found as follows:

$$
\begin{gathered}
I_{D}^{2} \cdot R_{D}>?<I_{D} \cdot V_{D} \\
I_{D}>?<\frac{V_{D}}{R_{D}} .
\end{gathered}
$$

The significance of (7) can be summarized as follows:

1) If $I_{D}>\frac{V_{D}}{R_{D}}$, then the resistive losses are higher, keep the doping and $R_{D}$ constant because decreasing $R_{D}$ means decreasing $B V$, which would decrease the voltage blocking capability of the device.

2) If $I_{D}<\frac{V_{D}}{R_{D}}$, then the $V_{D}$ losses are higher, decrease the doping so that $V_{D}$ will be smaller.

For different operation condition, the amount of current passing through each device and the voltage across them are calculated and the results are listed in Table II. According to Table II, $I_{D}$ varies between $47 \mathrm{~A}$ and $119 \mathrm{~A}$ for a $5 \mathrm{~kW}$ dc-dc converter in the HEV simulation, then applying the above criteria,

- If $47 A>\frac{V_{D}}{R_{D}}$, then the first criterion applies.

- If $119 A<\frac{V_{D}}{R_{D}}$, then the second criterion applies.

- If $47 A<\frac{V_{D}}{R_{D}}<119 A$, then it depends on how close the magnitude of the current is to the minimum or maximum values for the majority of the time. For example, if the average load is varying or constant and is in a range between 3.5 and $5 \mathrm{~kW}$, then the current is closer to the upper limit and the second criterion applies. If, on the other hand, the average load is in a range between 2 and $3.5 \mathrm{~kW}$, then the current is closer to the lower limit and the first criterion applies.

This criteria presented here, can be applied to almost any dc-dc converter using $\mathrm{SiC}$ devices.

\section{B. Switching Loss Parameters}

The diode switching losses occur due to the reverse recovery of the diode, which is caused by the stored charge in the depletion region. Schottky diodes are majority carrier devices, so they do not have stored charge. However, they display a characteristic similar to reverse recovery due to the ringing of the parasitics and the internal pn junction caused by the p-rings. The p-rings are used to reduce the large reverse leakage currents.

For Schottky diodes, the switching losses can be reduced either by reducing the parasitic elements or improving the reverse recovery characteristics of the pn junction formed by the p-rings.

A diode switching loss expression has been derived in [5] using Fig. 6:

$$
P_{r r}=f_{c} \frac{V_{R}}{2 S} \frac{\S d I_{F}}{(0) d t} \cdot \frac{\S S t_{r r}}{\AA} \cdot{ }^{2}
$$

where $f_{c}$ is the switching frequency, $V_{R}$ is the reverse blocking voltage, $I_{F}$ is the forward diode current, $S$ is the snappiness factor, and $t_{r r}$ is the reverse recovery time.

TABLE II

MAXimum DeVice Voltage AND CURRENTS For DifFERENT LOAD POWER AND INPUT VOLTAGE CONDITIONS

\begin{tabular}{cccccc}
\hline $\begin{array}{c}\boldsymbol{P}_{\text {out }} \\
(\mathbf{k W})\end{array}$ & $\begin{array}{c}\boldsymbol{V}_{\boldsymbol{d c}} \\
(\mathbf{V})\end{array}$ & $\begin{array}{c}\boldsymbol{V}_{\text {MOSFET }} \\
(\mathbf{V})\end{array}$ & $\begin{array}{c}\boldsymbol{I}_{\text {MOSFET }} \\
(\mathbf{A})\end{array}$ & $\begin{array}{c}\boldsymbol{V}_{\text {DIODE }} \\
(\mathbf{V})\end{array}$ & $\begin{array}{c}\boldsymbol{I}_{\text {DIODE }} \\
(\mathbf{A})\end{array}$ \\
\hline 2 & 300 & 300 & 6.67 & 84 & 47 \\
2 & 450 & 450 & 4.44 & 84 & 47 \\
5 & 300 & 300 & 16.67 & 84 & 119 \\
5 & 450 & 450 & 11.11 & 84 & 119 \\
\hline
\end{tabular}




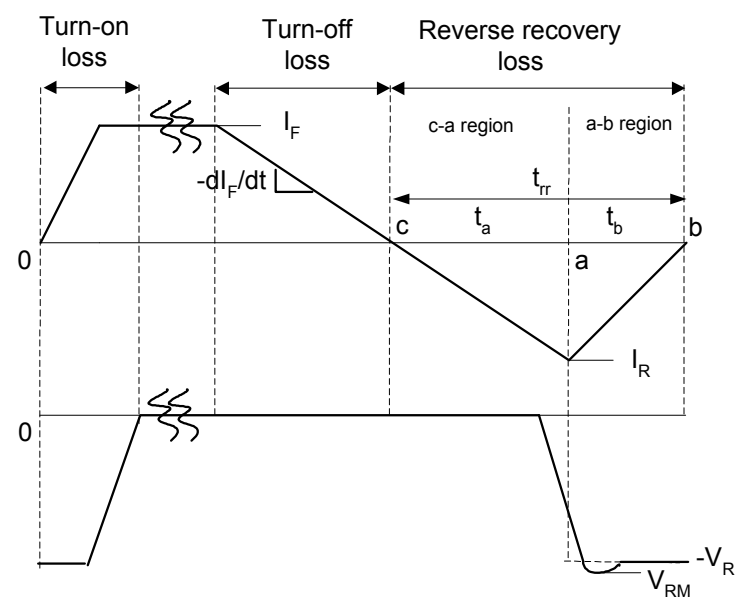

Fig. 6. Typical diode switching waveform.

In this expression all the parameters except $S$ and $t_{r r}$ are circuit dependent. These two parameters can be expressed [9] in other device parameters for a pn diode as follows,

$$
\begin{aligned}
& S=\frac{2 W_{d}}{b}-1 \\
& t_{r r}=\frac{2 b W_{d}}{D_{n}}
\end{aligned}
$$

where $D_{n}$ is the electron diffusion constant $\left(D_{n}=\frac{k T}{q} \mu_{n}\right), W_{d}$ is the width of the drift region, and $b$ is a distance in the drift region measured from the $p^{+} n^{-}$junction $b=\frac{2 q A D_{n}\left[n(0)-n^{*}\right]}{I_{F}}$ as shown in Fig. 7, $n(0)$ is the carrier density at the $p^{+} n^{-}$junction when the diode is on, and $n^{*}$ is the average carrier concentration in the $n^{-}$region.

Gathering the $S$ and $t_{r r}$ related terms in (8) and inserting (9) and (10), the following is obtained:

$$
\begin{aligned}
\frac{1}{S} \frac{\S S t_{r r}}{@ S+1} \cdot^{2} & =\frac{S t_{r r}^{2}}{(S+1)^{2}}=\frac{\frac{\S 2 W_{d}}{\complement b}-1_{\overrightarrow{1}} \frac{4 b^{2} W_{d}^{2}}{D_{n}^{2}}}{\frac{4 W_{d}^{2}}{b^{2}}} \\
& =\frac{2 W_{d}-b}{b} \frac{4 b^{4}}{D_{n}^{2}}=\frac{\left(2 W_{d}-b\right) b^{3}}{D_{n}^{2}}
\end{aligned}
$$

Therefore, decreasing $b$ and/or $W_{d}$ can decrease the switching losses and $b$ can be decreased by decreasing the area and/or $[n(0)-n *]$.

Note that the conclusions here also apply to the dc-dc converter, because (8) represents a switching cycle independent of the application.

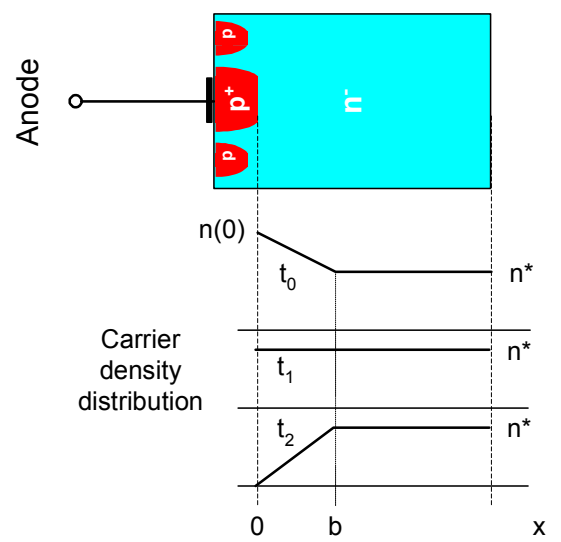

(a)

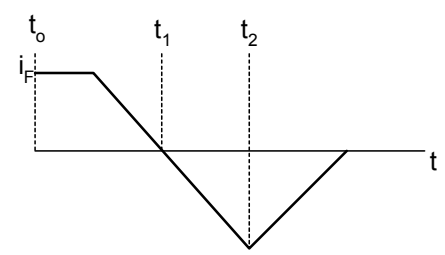

(b)

Fig. 7. Carrier distribution in a diode during turn-off (a) Linearized carrier density distribution of a diode at different time instants (b) Linearized turn-off current waveform of the diode.

\section{MOSFETS}

The following study will focus on the traction drive but the conclusions derived can also be applied to the dc-dc converter.

\section{A. Conduction Loss Parameters}

The conduction loss expression of a MOSFET in a traction drive has been derived in [5], and it is repeated below for convenience.

$$
P_{\text {cond }, Q 1}=I^{2} \cdot R_{D S, \text { on }} \cdot \frac{\S 1}{\mathbb{C} 8}+\frac{1}{3 \pi} M \cos \dot{\phi}_{\mathrm{I}}
$$

The only device related parameter in this expression is $R_{D S, \text { on }}$, which can be represented by other device parameters as follows

$R_{D S, o n} \approx R_{o n, s p}=\frac{4 B V^{2}}{\varepsilon_{s} \mu E_{c}^{3}}$

for a device with $1 \mathrm{~cm}^{2}$ area where $R_{o n, s p}$ is the specific on resistance of the MOSFET drift region and $\varepsilon_{s}, E_{c}$, and $\mu$ are material related constants.

Equation (13) is a rough estimate of a MOSFET resistance which also contains other resistive components like the channel resistance and the contact resistance. The drift resistance cannot be changed much; however, the channel and contact resistances can be lowered with more research. 


\section{B. Switching Loss Parameters}

The energy loss equation of a MOSFET has been shown in [5] as follows,

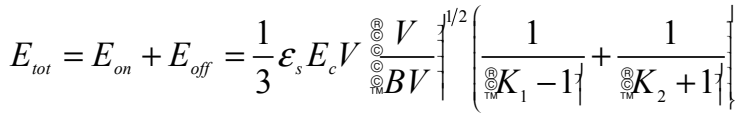

where $K_{1}=\frac{g_{m}\left(V_{G H}-V_{t h}\right)}{J}$ and $K_{2}=\frac{g_{m}\left(V_{t h}-V_{G L}\right)}{J}$,

$g_{m}$ is the transconductance,

$J$ is the current density,

$V_{G H}$ is highest gate voltage applied,

$V_{G L}$ is lowest gate voltage applied, and

$V_{t h}$ is the threshold voltage

If (14) is rearranged, (15) is obtained.

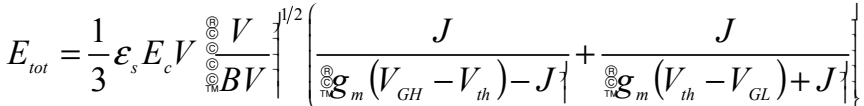

The most important parameter contributing to the MOSFET switching energy loss is the transconductance $g_{m}$. This parameter can be represented as follows [10],

$$
g_{m}=\mu \frac{w}{l} C_{o x} V_{D}=\mu \frac{w}{l} \stackrel{\S}{\S} \varepsilon_{o x} \frac{A_{o x}}{t_{o x}}, V_{D}
$$

where $\mu$ is the mobility, $w$ is the channel width,

$l$ is the channel length,

$C_{o x}$ is the oxide capacitance,

$V_{D}$ is the drain voltage,

$\mathcal{E}_{o x}$ is the oxide dielectric constant,

$t_{o x}$ is the oxide thickness, and

$A_{o x}$ is the oxide area.

In (16), $\mu$ and $\varepsilon_{o x}$ are material dependent; therefore, for a specific application, four device parameters affect the transconductance, $w, A_{o x}, l$, and $t_{o x}$. The first two of these parameters are directly proportional to $g_{m}$ and the others are indirectly proportional to it. From (16), the following statements can be derived:

- Decreasing $t_{o x}$ increases $g_{m}$ but $t_{o x}$ has to be of a minimum thickness to be able to support the rated gate voltage; therefore, it cannot be changed much.

- Decreasing $l$ increases $g_{m}$, but the value of $l$ is limited by the device processing technology.

- Increasing $A_{o x}$ increases $g_{m}$, but $A_{o x}$ depends on the device area; it cannot be arbitrarily increases without some difficulty.
- Increasing $w$ increases $g_{m}$. To increase $w$, the device area has to be increased proportionally.

As a summary, to decrease the MOSFET switching losses, $g_{m}$ needs to be increased. Increasing the device's area and consequently increasing $A_{o x}$ and $w$ seem to be the best method to do this.

\section{CONCLUSIONS}

In this paper, losses of the devices in a traction drive are investigated as functions of device parameters. Some modifications to device parameters are suggested to improve the losses in this drive. The next step is for device researchers to consider these suggestions and evaluate the viability of these modifications.

The interaction of device and power electronics researchers will be extremely useful in producing application specific power devices designed for optimum performance. This study is the first step to achieving this goal.

\section{REFERENCES}

[1] M. Bhatnagar and B. J. Baliga, "Comparison of 6H-SiC, 3C-SiC, and Si for power devices," IEEE Trans. on Electron Devices, vol. 40, no. 3, March 1993, pp. 645-655.

[2] K. Shenai, R. S. Scott, and B. J. Baliga, "Optimum semiconductors for high-power electronics," IEEE Transactions on Electron Devices, vol. 43, no. 9, Sept. 1989, pp. 1811-1823.

[3] A. Elasser, M. Kheraluwala, M. Ghezzo, R. Steigerwald, N. Krishnamurthy, J. Kretchmer, and T. P. Chow, "A comparative evaluation of new silicon carbide diodes and state-of-the-art silicon diodes for power electronic applications," IEEE IAS Annual Meeting Conference Proceedings, 1999, pp. 341-345.

[4] A. R. Hefner, D. Berning, J. S. Lai, C. Liu, and R. Singh, "Silicon Carbide merged PiN Schottky diode switching characteristics and evaluation for power supply applications," Proceedings of the Annual Meeting of the IEEE Industry Applications Society, 2000, pp. 29482954

[5] B. Ozpineci, L. M. Tolbert, S. K. Islam, and Md. Hasanuzzaman, "Effects of silicon carbide (SiC) power devices on PWM inverter losses," The Annual Conference of the IEEE Industrial Electronics Society (IECON'01), 2001, pp. 1187-1192.

[6] B. Ozpineci, L. M. Tolbert, S. K. Islam, and F. Z. Peng, "Testing, characterization, and modeling of $\mathrm{SiC}$ diodes for transportation applications, " IEEE Power Electronics Specialists Conference (PESC'02), June 23-27, 2002.

[7] B. Ozpineci, L. M. Tolbert, S. K. Islam, and Md. Hasanuzzaman, "System impact of silicon carbide ( $\mathrm{SiC})$ power devices," International Journal of High Speed Electronics and Systems, in press

[8] B. Ozpineci, System impact of silicon carbide power electronics on hybrid electric vehicle applications, August 2002

[9] B. J. Baliga, Power Semiconductor Devices, PWS Publishing Company, Boston, 1996.

[10] D. A. Grant and J. Gowar, Power MOSFETS-Theory and Applications, John Wiley \& Sons, New York, 1989. 